La Revue

des Droits

de l'Homme

\section{La Revue des droits de l'homme}

Revue du Centre de recherches et d'études sur les droits fondamentaux

Actualités Droits-Libertés | 2014

\title{
Une première application paradoxale mais ambitieuse du régime de protection des fonctionnaires lanceurs d'alerte
}

Lanceurs d'alerte (Liberté d'expression)

Jean-Philippe Foegle

\section{OpenEdition}

Journals

Édition électronique

URL : http://journals.openedition.org/revdh/863

DOI : $10.4000 /$ revdh.863

ISSN : 2264-119X

Éditeur

Centre de recherches et d'études sur les droits fondamentaux

Référence électronique

Jean-Philippe Foegle, « Une première application paradoxale mais ambitieuse du régime de protection des fonctionnaires lanceurs d'alerte », La Revue des droits de l'homme [En ligne], Actualités Droits-

Libertés, mis en ligne le 20 août 2014, consulté le 30 avril 2019. URL : http://journals.openedition.org/ revdh/863; DOI : 10.4000/revdh.863

Ce document a été généré automatiquement le 30 avril 2019.

Tous droits réservés 


\section{Une première application paradoxale mais ambitieuse du régime de protection des fonctionnaires lanceurs d'alerte}

Lanceurs d'alerte (Liberté d'expression)

Jean-Philippe Foegle

1 Si, comme l'écrivait de belle manière l'écrivain Albert Camus, «les chemins familiers tracés dans les ciels d'étés [peuvent] mener aussi bien aux prisons qu'aux sommeils innocents » (Albert Camus, "L'étranger", partie II, chap. III, p. 147), la lecture de l'arrêt du Tribunal Administratif de Cergy-Pontoise ne saura évoquer ni prison (intellectuelle), ni sommeil (dogmatique). Bien au contraire, celui-ci semble démontrer avec éclat que l'habituelle et attendue torpeur estivale ne fait obstacle ni aux innovations juridiques, ni à la créativité juridictionnelle.

2 En l'espèce, la requérante, directrice d'un Office Public d'Habitat, avait dénoncé, en application de l'article $40 \mathrm{du}$ code de procédure pénale ${ }^{1}$, les manquements aux règles de passation des marchés publics commis l'un de ses subordonnés, et par le président de l'office. Cette dénonciation avait notamment conduit le Tribunal correctionnel de Nanterre à déclarer ce dernier coupable des délits d'atteinte à la liberté d'accès ou à l'égalité des candidats dans les marchés publics et de prise illégale d'intérêts. Ayant été révoquée de ses fonctions pour motif disciplinaire, la requérante avait contesté la décision de révocation prise par sa hiérarchie devant le Tribunal Administratif de CergyPontoise puis devant la Cour Administrative d'Appel de Versailles qui avait annulé la décision litigieuse et enjoint à l'office de la réintégrer.

En réponse à sa demande de réintégration sur le poste de directeur général qu'elle occupait auparavant, le président de l'établissement public avait invité la requérante à présenter sa candidature à ce poste afin de la faire examiner, par le conseil d'administration de l'office. Le président de l'office avait alors présenté la candidature de la requérante de manière sciemment inexacte en indiquant notamment aux membres du 
conseil d'administration que la Cour administrative d'appel de Versailles avait reconnu que cette dernière avait commis des fautes graves dans la gestion de l'office. Par suite, le conseil d'administration de l'office avait alors rejeté la demande de réintégration formulée par la requérante, ce que celle-ci a par la suite contesté devant le Tribunal Administratif de Cergy-Pontoise.

4 Faisant droit à l'argumentation de la requérante, le tribunal apporte, par sa décision, une pierre supplémentaire à l'édifice chancelant que constitue le statut protecteur des lanceurs d'alerte de la fonction publique.

Qualifiant de détournement de pouvoir une mesure fondée en apparence sur les qualités professionnelles d'un fonctionnaire mais visant en réalité à sanctionner celui-ci en raison de la qualité de «lanceur d'alerte ", le jugement démontre ainsi de manière exemplaire comment certains mécanismes traditionnels $\mathrm{du}$ droit administratif peuvent potentiellement venir au secours des lanceurs d'alerte de la fonction publique $\left(1^{\circ}\right)$.

6 Mais, surtout, les juges du Tribunal font application, de manière prétorienne et (presque)superfétatoire mais également de manière plus ambitieuse, une application par anticipation du régime protecteur prévu par la loi n 2013-1117 du 6 décembre 2013 en consacrant un « principe général » du droit interdisant les mesures prises à l'égard d'un fonctionnaire pour avoir relaté ou témoigné, de bonne foi, de faits constitutifs d'un délit ou d'un crime dont il aurait eu connaissance dans l'exercice de ses fonctions $\left(2^{\circ}\right)$. Toutefois, la décision révèle également, malgré elle, une insuffisance et une incertitude quand à la portée du nouveau régime de protection des fonctionnaires lanceurs d'alerte ( $\left.3^{\circ}\right)$.

\section{$1 \%$ - Le détournement de pouvoir au secours des lanceurs d'alerte?}

7 Le détournement de pouvoir, défini par la doctrine administrative classique comme «le fait d'une autorité administrative qui, tout en accomplissant un acte de sa compétence, tout en observant les formalités prescrites, tout en ne commettant aucune violation formelle de la loi, use de son pouvoir pour des motifs autres que ceux en vue desquels ce pouvoir lui a été conféré, c'est-àdire autres que la sauvegarde de l'intérêt général et le bien du service $»^{2}$ se caractérise plus généralement et au-delà de toute idée de "moralité», comme l'utilisation par l'administration de prérogatives légalement instituées en vue de poursuivre un but illégal ou, à tout le moins, étranger à la poursuite de l'intérêt général. Le détournement de pouvoir peut-être caractérisé tant par les pièces du dossier ${ }^{3}$, des présomptions suffisamment fortes et des indices suffisamment sérieux ${ }^{4}$ et des allégations suffisamment précises dès lors que celles-ci ne sont pas contredites par les pièces du dossier ${ }^{5}$. L'on trouve des illustrations contentieuses du principe dans de nombreux domaines, et en particulier dans le domaine de la police municipale.

8 En l'espèce, la reconnaissance d'un détournement de pouvoir ne semblait pas particulièrement problématique. Le jugement indique en effet que le président de l'office avait « présenté la requérante de manière sciemment inexacte, indiquant aux membres du conseil d'administration que la Cour administrative d'appel de Versailles avait reconnu que Mme X. avait commis des fautes graves dans la gestion de l'office, » [...] alors que, par son arrêt $n^{\circ} 08 \mathrm{VE04005,}$ cette juridiction n'a reconnu comme fautive qu'un fait mineur » et, qu'au surplus, la mauvaise entente entre le président de l'office et la requérante n'était pas imputable à la 
requérante mais à la dénonciation par celle-ci «des infractions pénales qu'elle avait constatées». Presque évidente au vu des faits de l'espèce, la reconnaissance d'un détournement de pouvoir ne serait-elle pas exemplaire, et de nature à renouveler l'approche du contentieux des mesures discriminatoires prises à l'encontre des fonctionnaires lanceurs d'alerte, voire, surtout, d'éventuels usagers lanceurs d'alerte? En effet, là où le droit d'alerte dans la fonction publique convoque la figure du " fonctionnaire-citoyen ", le droit d'alerte de manière plus général convoque conceptuellement la vigilance de tous les citoyens. Ainsi, si - comme le soulignent les auteurs d'un rapport du conseil de l'Europe sur le sujet - l'exclusion des consommateurs et des usagers des lois de protection des « lanceurs d'alerte » est quelque peu arbitraire, les mécanismes classiques du droit administratif pourraient pallier à cette insuffisance

\section{$2 \%$ - Une consécration prétorienne d'un « principe général » protecteur des lanceurs d'alerte}

Mais l'apport réel de la décision commentée est ailleurs. C'est avant tout la consécration prétorienne d'un principe général du droit protecteur des lanceurs d'alerte qui fait, en effet, tout l'intérêt du raisonnement des juges du Tribunal. Ceux-ci considèrent en effet, dans l'espèce commentée, qu' « en vertu d'un principe général, dont s'inspirent au demeurant les dispositions de l'article 6 ter A de la loi du 13 janvier 1983 issues de la loi du no 2013-1117 du 6 décembre 2013 et donc non applicables au présent litige, aucune mesure concernant notamment le recrutement ne peut être prise à l'égard d'un fonctionnaire pour avoir relaté ou témoigné, de bonne foi, de faits constitutifs d'un délit ou d'un crime dont il aurait eu connaissance dans l'exercice de ses fonctions"

10 Certes, il n'a pas fallu attendre la présente décision pour qu'émergent des dispositifs protecteurs des lanceurs d'alerte. Sous l'influence du droit de l'Union européenne ${ }^{7}$, le statut général prévoit ainsi qu'« aucune mesure concernant notamment le recrutement, la titularisation, la formation, la notation, la discipline, la promotion, l'affectation et la mutation ne peut être prise à l'égard d'un fonctionnaire " qui a témoigné ou relaté d'agissements constitutifs soit de distinction, directe ou indirecte, en raison de certains motifs de discrimination prohibés ${ }^{8}$, soit de distinctions entre hommes et femmes ${ }^{9}$, soit de faits de harcèlement sexue ${ }^{10}$, soit de faits de harcèlement moral ${ }^{11}$.Ce n'est toutefois qu'à compter de la loi n 2013-1117 du 6 décembre 2013 qu'a été instituée une protection générale des lanceurs d'alerte incluant l'ensemble des agents publics, par un article 6 ter A au sein de la loi de 1983 portant droit et obligations des fonctionnaires. Calqué sur le modèle des dispositifs suscités contre les agissements discriminatoires ou les faits de harcèlement, cet article 6 ter A prévoit qu'« aucune mesure concernant notamment le recrutement, la titularisation, la formation, la notation, la discipline, la promotion, l'affectation et la mutation » ne peut être prise à l'égard d'un fonctionnaire ou d'un agent non titulaire de droit public, qui aurait «relaté ou témoigné, de bonne foi, de faits constitutifs d'un délit ou d'un crime dont il aurait eu connaissance dans l'exercice de ses fonctions $»^{12}$.

11 Cette nouvelle législation, dont les apports mais également (et surtout) les limites ont été soulignées au sein de cette revue ${ }^{13} n^{\prime}$ était toutefois pas applicable au litige, puisque la décision attaquée avait été rendue, précisément, avant l'intervention de la loi. Or, cette nouvelle protection apparaît essentielle pour permettre de concilier l'article 40 du code 
de procédure pénale, qui apparaît de manière croissante comme un pivot de la lutte anticorruption, et la situation de dépendance hiérarchique dans laquelle sont placés les agents publics. Ceux-ci se trouvaient, avant l'intervention de la loi, face à un dilemme lorsqu'ils ont connaissance de faits délictuels ou criminels dans le cadre de leur service : faut-il, en toutes circonstances, «faire preuve de discrétion professionnelle» ou, au contraire, donner systématiquement « avis sans délai au procureur de la République » de faits délictueux?

Cette contrainte juridique n'a toutefois pas empêché le tribunal de faire application, par anticipation, du régime protecteur des lanceurs d'alerte de la fonction publique, en créant de manière prétorienne un "principe général» dont s'inspireraient les dispositions du nouvel article 6ter du statut général des fonctionnaires. Si l'on peut douter $\mathrm{du}$ fait que le législateur se soit borné à reconnaître un principe juridique protecteur pré-existant, et si l'on peut également douter que l'autorité d'un jugement de tribunal administratif soit de nature à faire émerger un "principe général», cette décision pragmatique devrait, sans doute, être saluée.

Sur le plan théorique, il ne paraît pas absurde de reconnaître comme un principe général l'interdiction de prendre des mesures de rétorsion à l'égard des fonctionnaires dénonçant des faits illégaux. En effet, si l'on raisonne par analogie, la multiplication des dispositifs prévoyant des "obligations de dénoncer» et des protections pour les dénonciateurs (citées supra) ainsi que l'existence un droit (limité) de désobéir inscrit à l'article 28 de la loi de 1983 tirant son origine dans une jurisprudence ancienne du conseil d'Etat y inciterait ${ }^{14}$.

Plus largement, la dénonciation d'actes illégaux par des agents publics apparaissant comme un acte citoyen par excellence, il ne semble pas absurde de considérer que l'architecture générale des lois «Le Pors» de 1983-1984 portant statut général de la fonction publique, qui avaient voulu instituer un "fonctionnaire-citoyen $»^{15}$, puissent impliquer l'existence d'un tel principe. L'on pourrait également, d'un point de vue plus théorique, trouver un fondement à un tel principe dans l'article 2 de la déclaration des droits de l'homme et du citoyen de 1789 qui instaure un "droit de résistance à l'oppression " ${ }^{16}$ : l'on sait en effet que ce droit a une valeur constitutionnelle ${ }^{17}$, bien qu'aucune application concrète n'en ait été faite.

Or, comme le souligne Geneviève Koubi ${ }^{18}$, s'il est conçu comme un droit processuel, le droit de résistance à l'oppression » pourrait alors « acquérir son plein exercice dans le cadre dit du "recours pour excès de pouvoir". "et, " défait de sa qualité révolutionnaire, de sa connotation politique, de son assimilation à la rébellion » par sa consécration juridictionnelle, celui-ci pourrait s'exprimer dans la référence à la légalité. Celle-ci « attesterait ainsi du caractère fondamental du droit de résistance à l'oppression comme un "droit au respect du droit" ». Le « droit de résistance à l'oppression pourrait ainsi, hors de toute référence à un droit naturel, trouver une application pratique, processuelle, en protégeant les agents publics réclamant leur "droit à la légalité" ".

Surtout, au delà de ces éléments théoriques, la solution doit être saluée pour son pragmatisme. Celle-ci permettrait en effet, de manière transitoire, de faire bénéficier les fonctionnaires lanceurs d'alerte des protections récemment instituées quand bien même le litige qui les oppose à leur administration serait intervenu avant la publication de la loi du 6 décembre 2013. 


\section{$3 \%$ - Une décision révélant une incertitude et une insuffisance du régime protecteur des fonctionnaires lanceurs d'alerte}

La présente décision, révèle toutefois, malgré elle, une incertitude et une insuffisance s'agissant du régime de protection instauré par la loi du 6 décembre 2013. Le caractère souple de la preuve dans le cadre du détournement de pouvoir et le fait que le raisonnement du juge lie immédiatement la reconnaissance du détournement de pouvoir avec le régime instauré par le nouvel article 6ter (voir partie 2) laisse à penser qu'en réalité, les juges ont ici fait application - par la médiation d'autres mécanismes - du nouveau régime protecteur des lanceurs d'alerte de la fonction publique. Or, une incertitude règne, notamment sur l'appréciation de la « bonne foi » exigée du lanceur d'alerte. Implicitement, les juges reconnaissent ici la « bonne foi » de la requérante qui peut se prévaloir d'une décision de justice condamnant son supérieur hiérarchique, et la faiblesse des motifs invoqués pour que soit refusée sa réintégration paraissaient bien faibles en comparaison de ce simple fait.

Mais qu'en serait-il dans le cas où un agent public lanceur d'alerte signalerait un délit ou un crime par d'autres biais que l'article 40-2 du Code de Procédure Pénale, en passant par la voie de la presse ou d'une autorité indépendante, par exemple ? En ce cas, la « bonne foi » de l'agent public serait plus douteuse, et l'employeur de l'agent public pourrait se prévaloir d'éléments de nature à justifier sa décision : la rupture de l'obligation de réserve, voire du secret professionnel dans certains cas?

Là est sans doute le paradoxe du nouveau régime protecteur des « lanceurs d'alerte » de la fonction publique : laissant coexister un « droit » d'alerter représenté par l'article 6ter du statut général de la fonction publique et un " devoir » de dénoncer institué par l'article 40-2 du Code de procédure pénale, celui-ci n'entoure pas le droit d'alerter de garanties suffisantes. Car si la dénonciation au procureur de faits délictueux est une obligation à la charge de l'agent public et qu'elle fait bien partie des hypothèses légales permettant de se délier de l'obligation de réserve, il n'en va pas de même s'agissant du « droit » d'alerter d'autres autorités qui semble implicitement ouvert par l'article 6ter du statut. L'exercice de celui-ci se heurterait directement à l'obligation de réserve de l'agent public ${ }^{19}$. Il faudrait alors appliquer la grille de lecture proposée par Cour européenne des droits de l'homme dans son arrêt Guja $^{20}$ et s'intéresser au contenu et aux modalités de la dénonciation. Exercice ô combien périlleux !

Les juges devraient alors examiner si l'agent public a opéré la divulgation « d'abord auprès de son supérieur ou d'une autre autorité ou instance compétente » et, en cas d'appel à la presse, " examiner si l'intéressé disposait d'autres moyens effectifs de faire porter remède à la situation qu'il jugeait critiquable » (\$73). Devrait par la suite être pris en compte «le poids respectif du dommage que la divulgation litigieuse risquait de causer à l'autorité publique et de l'intérêt que le public pouvait avoir à obtenir cette divulgation ", ainsi que l'authenticité des informations divulguées (\$76) et surtout, de manière hautement problématique, que cette révélation n'a pas été motivée " par un grief ou une animosité personnels ou encore par la perspective d'un avantage personnel, notamment un gain pécuniaire [...] (\$77). On saisit l'étroitesse de la marge de manœuvre du lanceur d'alerte qui fait usage de son supposé « droit d'alerte ». 
21 La décision révèle en outre et encore une fois malgré elle, une insuffisance du nouveau régime de protecteur des lanceurs d'alerte. En effet, le tribunal n'ordonne ici qu'un " réexamen " (article 2 du dispositif du jugement) de la situation de la requérante, et il n'en serait pas allé différemment si la loi du 6 décembre avait été applicable. En effet, celle-ci ne prévoie pas la réintégration de plein droit de l'agent public victime d'agissements discriminatoires, insuffisance immédiatement soulignée par Transparency International ${ }^{21}$ . Preuve que le "malentendu francais » en matière de protection des fonctionnaires lanceurs d'alerte n'est pas encore totalement dissipéz ${ }^{2}$.

Bien qu'il ne fasse pas stricto sensu application de la loi du 6 décembre 2013, le jugement commenté offre une première mise en application jurisprudentielle encourageante de celui-ci. En outre, celui-ci démontre que le mécanisme du "détournement de pouvoir » pourrait sans doute être utilement utilisé dans le cadre de la défense des lanceurs d'alerte, notamment dans l'hypothèse où le statut protecteur récemment adopté -qui n'est nullement complet- ne leur serait pas applicable. Plus largement, la présente décision confirme que le dispositif prévu par l'article 40 al. 2 du code de procédure pénale constitue bien désormais un « pivot » de la lutte contre la corruption ${ }^{23}$ et que, lentement mais sûrement, l'intervention de 5 lois protectrices des lanceurs d'alerte depuis 2008 tend à créer une culture juridique ouverte à l'alerte. Celle-ci, on l'a constaté, est toutefois encore incomplète.

Au-delà, l'inventivité dont ont fait preuve les juges du tribunal administratif de CergyPontoise peut permettre d'imaginer d'éventuelles voies nouvelles s'agissant de la protection des lanceurs d'alerte de la fonction publique. Ne pourrait-on pas, en effet, envisager qu'une juridiction suprême consacre, plus largement, un principe plus général protecteur de tous les agents publics dénonçant des faits contraires à l'intérêt public et non plus simplement des faits illégaux ? L'évolution général du "droit à la désobéissance » des fonctionnaires, qui « montre très nettement que le caractère manifestement illégal de l'acte s'efface au profit de l'atteinte grave qu'il porte à un intérêt public pour déterminer la légalité de l'usage de la désobéissance ${ }^{24}$ pourrait inciter à le penser : si une atteinte grave à un intérêt public légitime la désobéissance, ne légitimerait-il pas a fortiori la protection de ceux qui dénoncent ces mêmes actes?

Loin de la consécration d'un tel principe, la jurisprudence récente du conseil d'Etat semble extrêmement timide, pour ne pas dire timorée, en la matière, bien que la réflexion sur les «lanceurs d'alerte» semble récemment prendre une plus grande importance aux yeux de cette institution ${ }^{25}$. Ce sera, sans doute, au législateur que reviendra la tâche de compléter un statut qui, par son caractère éclaté et partiel, est encore bien loin d'atteindre l'« approche globale » du phénomène du lancement d'alerte qui a fait le succès de la notion dans certains pays Anglo-Saxons. ${ }^{26}$

TA Cergy-Pontoise, 7e Ch., 15 juillet 2014, M.H., Req. No 1110539 
Les Lettres « Actualités Droits-Libertés » (ADL) du CREDOF (pour s'y abonner) sont accessibles sur le site de la Revue des Droits de l'Homme (RevDH) - Contact

\section{NOTES}

1. L'article 40 alinéa 2 du code de procédure pénale - décliné dans d'autres codes en diverses dispositions miroir, prévoit que «toute autorité constituée, tout officier public ou fonctionnaire qui, dans l'exercice de ses fonctions, acquiert la connaissance d'un crime ou d'un délit est tenu d'en donner avis sans délai au procureur de la République et de transmettre à ce magistrat tous les renseignements, procèsverbaux et actes qui y sont relatifs "; il s'agit de l'un des dispositifs essentiels d' " alerte éthique » au sein de la fonction publique française. Sur la portée de cet article, v. CE, 15 mars 1996, Guigon, n - 146326, Rec. CE 1996, tables, p. 1109 ; V. également, G. Chalond, « Le fonctionnaire et l'article 40 du Code de procédure pénale : nature et portée de l'obligation de dénoncer ", AJFP nov. 2003, p. 31.

2. Maurice Hauriou, Précis de droit administratif, Paris, Sirey, 1927, 11e éd., p. 419.

3. CE, 19 février 1909, Abbé Olivier.

4. CE, Sect. 3 mars 1961.

5. CE ; 26 octobre 1960, Rioux.

6. Paul Stephenson, Michael Levi, «La protection des donneurs d'alerte », Rapport d'étude sur la faisabilité d'un instrument juridique sur la protection des employés qui divulguent des informations dans l'intérêt public, Conseil de l'Europe, CDCJ(2012)9FIN, Strasbourg, décembre 2012, p. 40.

7. Directive 2000/78/CE, du 27 novembre 2000, portant création d'un cadre général en faveur de l'égalité de traitement en matière d'emploi et de travai

8. Article 6 loi de 1983 modifié par l'article 4 de la loi nº 2012-954 du 6 août 2012

9. Article 6bis de la loi du 13 juillet 1983

10. Article 6 ter deLEtt la loi de 1983 modifié par l'article 8 de la loi $n^{\circ} 2012-954$ du 6 août 2012)

11. Article 6 quinquiès de la loi de 1983 modifié par l'article 6 de la loi n² 2005-843 du 26 juillet 2005

12. Article 26 de la loi $n^{\circ} 83-634$ du 13 juillet 1983 portant droits et obligations des fonctionnaires

13. Jean-Philippe Foegle et Serge Slama, «Refus de transmission d'une QPC sur la protection des fonctionnaires lanceurs d'alerte ", La Revue des droits de l'homme, 14 mars 2014 ; Anna Billard, Marc Duranton, Jean-Philippe Foegle et Tristan Martin-Teodorczyk, "Le "milieu du gué" de la protection législative des lanceurs d'alerte », La Revue des droits de l'homme, 20 mai 2014.

14. CE, 10 nov. 1944, Langneur

15. Geneviève Koubi, "Réflexions sur la qualité de fonctionnaire citoyen ", in Les Petites Affiches, 29 juillet 1988, p. 2. 
16. « Le but de toute association politique est la conservation des droits naturels et imprescriptibles de l'Homme. Ces droits sont la liberté, la propriété, la sûreté, et la résistance à l'oppression ».

17. Cons. constit., décision n 81-132 DC du 16 janvier 1982, Loi de nationalisation, cons. 13.

18. Geneviève Koubi, «Penser le droit de résistance à l'oppression dans les sociétés démocratiques contemporaines ", in Obéir et désobéir. Le citoyen face à la loi, Brussels: Éditions de l'Université de Bruxelles, 2000.

19. Stephen Pringault, «L'obligation de réserve des agents publics face au devoir de dénonciation d'infractions pénales. Une inadaptation du droit français à la problématique du whistleblowing ", DA, 2012, étude 8.

20. CEDH, 12 février 2008, Guja c. Moldavie, Req. n 14277/04, \$71 ; Voir, sur ce sujet : JUNOD (V.), « La liberté d'expression du whistleblower », RTDH, 2009, p. 227 ;JUNOD (V.), « Lancer l'alerte : quoi de neuf depuis Guja ? ", RTDH, n 98, 2014, p. 459.

21. L'on signalera à cet égard la publication récente et bienvenue d'un « Guide pratique à l'usage du lanceur d'alerte français ».

22. Nicole-Marie Meyer, «Alerte éthique et fonction publique : la fin d'un malentendu français? ", Revue des juristes de Sciences Po, n 9, 2014, p. 158.

23. ROMANET (L.), «Le dispositif d'alerte éthique de l'article 40 alinéa 2 du code de procédure pénale : un «instrument juridique pivot » de la lutte contre la corruption publique ?", La revue du Grasco, $\mathrm{n}^{\circ}$ 7, novembre 2013

24. V. Antony Taillefait, « Obligations des agents publics », JurisClasseur Administratif, fasc. 183, 2013

25. V. notamment, «Quelle déontologie pour les hauts fonctionnaires?», Intervention de JeanMarc Sauvé, vice-président du Conseil d'État, à l'Ecole nationale d'administration le 27 mars 2013.

26. V. Voir, notamment : Brown, A. J., "Flying Foxes and Freedom of Speech: Statutory Recognition of Public Whistleblowing in Australia.",Whistleblowing and Democratic Values (2011): 86 ; Elletta Sangrey Callahan, Terry Morehead Dworkin, and David Lewis, "Whistleblowing: Australian, UK, and US Approaches to Disclosure in the Public Interest.", Va. J. Int'l L. 44 (2003): 879.

\section{RÉSUMÉS}

Par une décision en date du 15 juillet 2014, le Tribunal Administratif de Cergy-Pontoise a fait droit aux prétentions d'une requérante "lanceure d'alerte " qui contestait une décision discriminatoire prise par sa hiérarchie. Offrant une illustration intéressante de la façon dont des mécanismes classiques du droit administratif - ici, celui du détournement de pouvoir - peuvent venir au secours des cassandres de la fonction publique, la décision du tribunal est en outre particulièrement créative. En effet, celle-ci dégage, par anticipation, un "principe général du droit " interdisant à l'administration de prendre des mesures discriminatoires à l'encontre des fonctionnaires ayant dénoncé des faits délictueux commis par leurs subordonnés ou par leur hiérarchie. Première application encourageante, avant la lettre, du récent statut protecteur des «lanceurs d'alerte », la décision incite néanmoins à mesurer, en creux, l'étendue du chemin restant à parcourir pour permettre une protection adéquate des lanceurs d'alerte. 
AUTEUR

JEAN-PHILIPPE FOEGLE

Etudiant du Master 2 "Droit de l'Homme" de l'Université de Paris Ouest Nanterre La Défense 